IOS Press

\title{
Erratum
}

\section{The Relationship Between Anxiety and Alzheimer's Disease}

Mario F. Mendez

Pre-press 25 June 2021

[Journal of Alzheimer's Disease Reports, 5(1) (2021), 171-177, 10.3233/ADR-210294]

https://content.iospress.com/articles/journal-of-alzheimers-disease-reports/adr210294

When this article was first published the author's name was mistakenly written as "Menddez". This has been corrected to "Mendez" in the revised online version of the article (DOI: 10.3233/ADR-210294). 